\title{
Basic study of PPD for the next generation of IACTs
}

\author{
Yoshitaka Mizumura*, Kazuhito Kodani, Junko Kushida and Kyoshi Nishijima \\ Department of Physics, Tokai University \\ 1117 Kita-Kaname, Hiratsuka-City, Kanagawa 259-1292 JAPAN \\ E-mail: mizumuraetkikam.sp.u-tokai.ac.ip
}

\begin{abstract}
Now, the VHE $\gamma$-ray astronomy is moved from the detection era into the detailed physics era. Imaging Atmospheric Cherenkov Telescopes (IACTs) are demanded a higher sensitivity and a lower energy threshold, for discussions of astrophysics and astroparticle physics. For these purposes, we focus on the PPD which is a novel photon detector made up of multiple APD pixels operated in Geiger mode. We started the research for the possibility of applying PPDs to the next generation of IACTs. As a first step, we measure the response of $1 \mathrm{~mm} \times 1 \mathrm{~mm}$ size and $3 \mathrm{~mm} \times 3 \mathrm{~mm}$ size samples of PPD, and study the basic characteristics such as the gain, noise rate, cross talk probability and cross talk + after pulse probability. We confirmed these characteristics of $1 \mathrm{~mm} \times 1 \mathrm{~mm}$ size PPD are consistent with reported results by the other groups, and we verified the gain characteristics of $3 \mathrm{~mm} \times 3 \mathrm{~mm}$ size PPD are the same as those expected from $1 \mathrm{~mm} \times 1 \mathrm{~mm}$ size PPDs.
\end{abstract}

International Workshop on New Photon Detectors PD09

June 24-26 2009

Shinshu University Matsumoto Japan

\footnotetext{
* Speaker.
} 


\section{Introduction}

Now, the number of discovered VHE $\gamma$-ray objects exceeds 80, which means the VHE $\gamma$ ray astronomy is moved from the detection era into the detailed physics era. Imaging Atmospheric Cherenkov Telescopes (IACTs) are demanded a higher sensitivity and a lower energy threshold, for discussions of astrophysics and astroparticle physics[四][]. For existing IACTs, the photomultiplier tube (PMT) is used most commonly as a photon detector. However, PMTs require high operation voltage and have low quantum efficiency.

The PPD is a photon detector consisting of multiple Geiger mode APD pixels. It has a signal output, and when it detects photons, the signal from the PPD is proportional to the number of hit APD pixels. The most remarkable features of the PPD are fine photon-counting capability and higher photodetection efficiency. However, its size is too small for the astroparticle experiments, and there are problems that the dark counts, the optical cross talk, and the after pulses. Furthermore, the gain is very sensitive to the operating conditions such as bias voltage and temperature. These disadvantages should be improved for future astroparticle experiments.

The Multi-Pixel Photon Counter (MPPC) is one of such PPDs manufactured by Hamamatsu Photonics K.K. in Japan [四] [团]. Here we studied the basic properties of the $1 \mathrm{~mm} \times 1 \mathrm{~mm}$ and $3 \mathrm{~mm} \times 3 \mathrm{~mm}$ size MPPCs. The geometries of the tested MPPCs are summarized in Table $\mathbb{W}$. The results of our measurements are presented in this paper.

Table 1: Specifications of measured MPPCs

\begin{tabular}{ccccc}
\hline Serial ID & Type & Photosensitive area & Number of pixels & Pixel pitch $[\mu \mathrm{m}]$ \\
\hline 602 & S10362-11-050C & $1 \mathrm{~mm} \times 1 \mathrm{~mm}$ & 400 & 50 \\
441 & S10362-11-100C & $1 \mathrm{~mm} \times 1 \mathrm{~mm}$ & 100 & 100 \\
455 & S10362-33-050C & $3 \mathrm{~mm} \times 3 \mathrm{~mm}$ & 3600 & 50 \\
464 & S10362-33-100C & $3 \mathrm{~mm} \times 3 \mathrm{~mm}$ & 900 & 100 \\
\hline
\end{tabular}

\section{Measurement setup}

The measurements discussed here are gain and noise rate which depend on bias voltage and temperature. The MPPC is set in the light-tight constant-temperature unit where the temperature is controlled with accuracy of $\pm 1{ }^{\circ} \mathrm{C}$ between $-10^{\circ} \mathrm{C}$ and $25^{\circ} \mathrm{C}$. Bias voltage is controlled by a stabilized power supply with an accuracy of $\pm 0.1 \mathrm{~V}$ around $70 \mathrm{~V}$. When we get the ADC spectrum for measurement of the gain of the MPPC, it is illuminated with blue LED light controlled by a pulse mode function generator. The pulse duration per pulse is $8 \mathrm{~ns}$ and the repetition frequency is $3 \mathrm{kHz}$. The MPPC signal is amplified for $\sim 100$ times using an amplifier, and is digitized by a CAMAC ADC. The synchronized signal from the function generator is used to generate the trigger of ADC, and the gate width is set between $80 \mathrm{~ns}$ and $180 \mathrm{~ns}$ depending on the number of pixels. When we measure the dark count rate, a self-trigger signal is used.

\section{Results of Gain measurements}

Fig. $\mathbb{W}$ shows an example of ADC counts distribution of dark current with a tiny LED illumination. The first peak is pedestal, and the following peaks indicate the number of hit cells during gate width, which correspond to the number of photons as long as it is significantly smaller than 
the number of cells. The ADC spectrum is fitted by a multi-Gaussian function to find positions of peaks and to use the following analyses.

The distance between the pedestal peak and the first peak corresponds to the gain of MPPC. Then, the MPPC gain $G_{M P P C}$ can be expressed as

$$
G_{M P P C}=\frac{A D C_{d i s} \times C o n v}{e \times G_{a m p}}
$$

where $A D C_{d i s}$ is the average distance between adjacent peaks, Conv $=0.244 \mathrm{pC} /$ count is a conversion factor from ADC counts to charge, $G_{\text {amp }} \simeq 93.2$ is the amplifier gain, and $e$ is the electron charge. The determined gain for Serial ID $=602$ is plotted in Fig. $\square$ as a function of the bias voltage $V_{\text {bias }}$, where each fitted line corresponds to a given value of temperature.

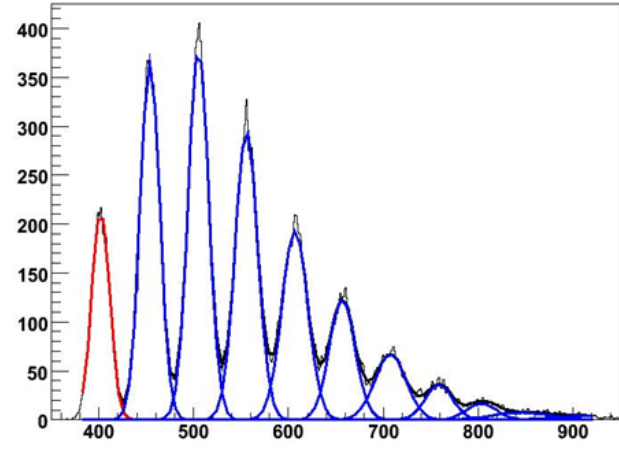

Figure 1: $A D C$ spectrum of the $1 \mathrm{~mm} \times 1 \mathrm{~mm}$ size MPPC (S10362-11-050C) with $10^{\circ} \mathrm{C}$ and $69.5 \mathrm{~V}$ triggered by pulsed light that fitted by multi-Gaussian convolved function. In this case, ten gaussians were used in the fitting.

The breakdown voltage $V_{\text {break }}$ is determined by extrapolating the fitted line in Fig. \ to the gain equal to zero. Fig. [] shows the breakdown voltage $V_{\text {break }}$ which linearly increase as the temperature becomes higher. The slope is $(5.4 \sim 6.0) \times 10^{-2} \mathrm{~V}^{\circ} \mathrm{C}^{-1}$.

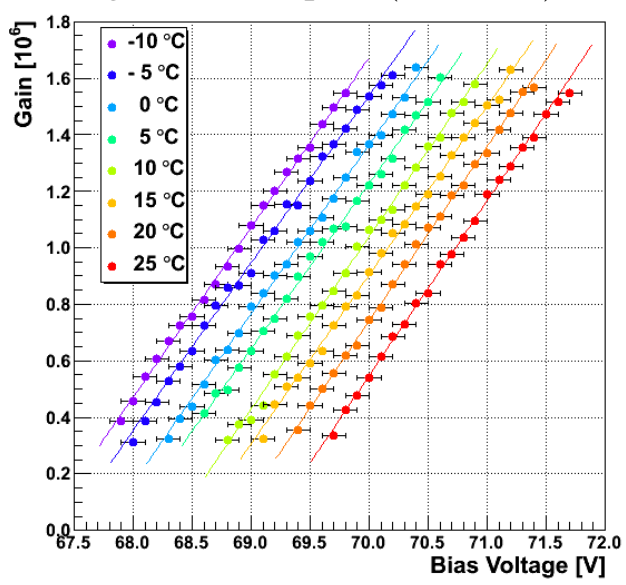

Figure 2: Bias voltage characteristic of gain of S10362-11-050C with temperature from $-10^{\circ} \mathrm{C}$ to $25^{\circ} \mathrm{C}$.

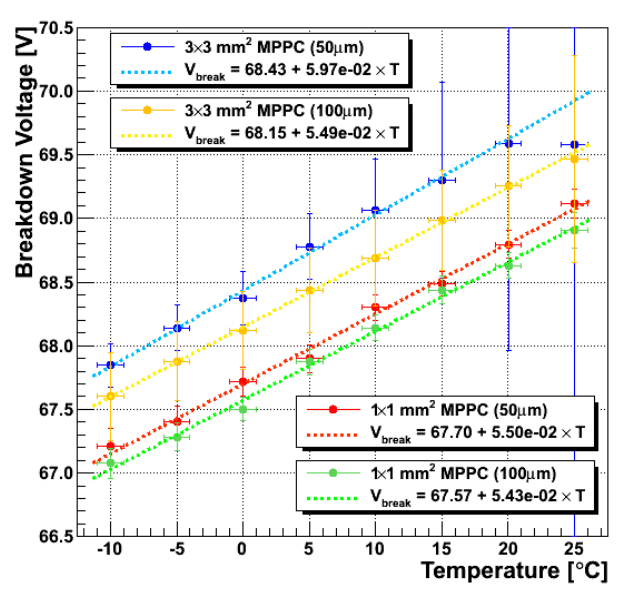

Figure 3: Temperature characteristics of breakdown voltage of four MPPCs.

From Fig. $\square$ and [1, the gain can be plotted as a function of over voltage $\Delta V=V_{\text {bias }}-V_{\text {break }}(T)$. The results including the other samples show that the over voltage dependence of the gain is linear,

Table 2: Gain slopes of over voltage characteristics and cell capacitances

\begin{tabular}{cccc}
\hline Serial ID & Gain slope $\left[\mathrm{V}^{-1}\right.$ ] & Cell capacitance [fC] & Terminal capacitance $[\mathrm{pC}]$ \\
\hline 602 & $5.97 \times 10^{5}$ & $95.5 \pm 0.04$ & $38.2 \pm 0.02$ \\
441 & $2.53 \times 10^{6}$ & $405.3 \pm 0.15$ & $40.5 \pm 0.02$ \\
455 & $5.57 \times 10^{5}$ & $89.1 \pm 0.08$ & $320.7 \pm 0.30$ \\
464 & $2.26 \times 10^{6}$ & $365.4 \pm 0.48$ & $328.9 \pm 0.43$ \\
\hline
\end{tabular}


and does not depend on the temperature. It depends on the pixel size, which corresponds to the capacitance of each pixel, because the gain is proportional to the capacitance, $G_{M P P C}=C_{p i x} \Delta V$, where $C_{p i x}$ is a pixel capacitance. The slopes and capacitances are summarized in Table $\square$.

For a given bias voltage, the gain linearly decreases with the temperature. The examples are shown in Fig. 田. The temperature coefficients of gain of $1 \mathrm{~mm} \times 1 \mathrm{~mm}$ size MPPC are $-3.21 \times 10^{4}\left[{ }^{\circ} \mathrm{C}^{-1}\right]$ for $50 \mu \mathrm{m}$ pitch and $-1.33 \times 10^{5}\left[{ }^{\circ} \mathrm{C}^{-1}\right]$ for $100 \mu \mathrm{m}$ pitch, and the same coefficients of $3 \mathrm{~mm} \times 3 \mathrm{~mm}$ size are $-3.13 \times 10^{4}\left[{ }^{\circ} \mathrm{C}^{-1}\right],-1.26 \times 10^{5}\left[{ }^{\circ} \mathrm{C}^{-1}\right]$, respectively. This means, in $10^{6}$ gain operation, $3^{\circ} \mathrm{C}$ variation affects to the gain by $\sim 10 \%$ and $\sim 40 \%$ for $50 \mu \mathrm{m}$ and $100 \mu \mathrm{m}$ pitch devices, respectively.

\section{Results of Noise measurements}

Dark count rates as a function of over voltage, which are taken at four fixed temperature of $-5^{\circ} \mathrm{C}$, $5^{\circ} \mathrm{C}, 15^{\circ} \mathrm{C}$ and $25^{\circ} \mathrm{C}$, for two levels of threshold, are

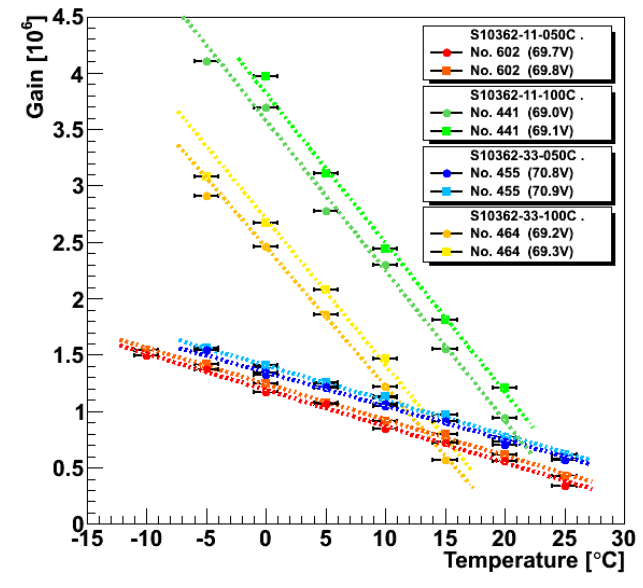

Figure 4: Temperature characteristics of gain with fixed bias voltage. shown in Fig. 5 and 6 , for the $1 \mathrm{~mm} \times 1 \mathrm{~mm}$ and the $3 \mathrm{~mm} \times 3 \mathrm{~mm}$ size samples, respectively. From these figures, we can see that the dark count rate increases at higher over voltage which corresponds to higher gain.

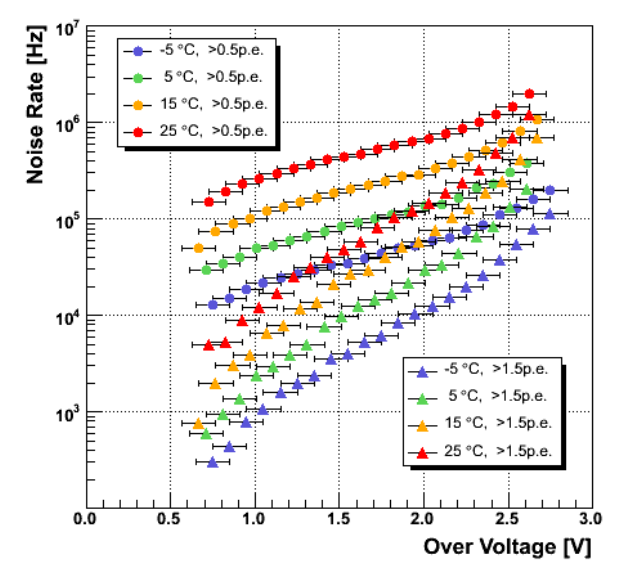

Figure 5: Over voltage characteristics of noise rate of S10362-11-050C with 0.5p.e. threshold level and 1.5p.e. threshold level, respectively.

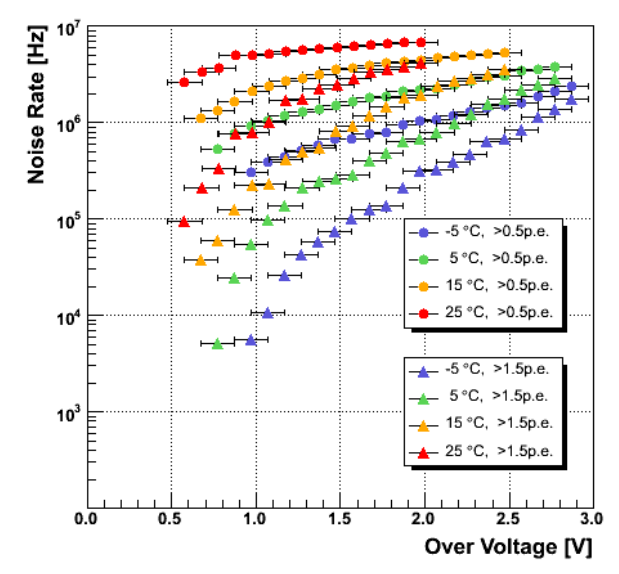

Figure 6: Over voltage characteristics of noise rate of S10362-33-050C with two levels of threshold, but here including the pile up effect.

Assuming the dark noise corresponding to the 2-pixel or more hits are dominantly produced by the cross talk effect, the ratio of dark count rates above 1.5 p.e. threshold and above 0.5 p.e. threshold gives an estimate of the cross talk probability. Fig. $\square$ shows the cross talk probability as a function of over voltage for two kinds of samples of $1 \mathrm{~mm}^{2}$ size MPPCs (green;100 $\mu \mathrm{m}$ pitch, red;50 $\mu \mathrm{m}$ pitch). The data points measured at four different temperatures between $-5^{\circ} \mathrm{C}$ and $25^{\circ} \mathrm{C}$ are plotted by the same symbol. As you can see, the over voltage dependences of the cross 
talk probability for the different temperature are identical, and the cross talk probability increases as the over voltage increases. At the fixed over voltage, the probability does not depend on the temperature. The over voltage characteristics of the cross talk probability is well fitted by the combination of the linear function and the exponential function.

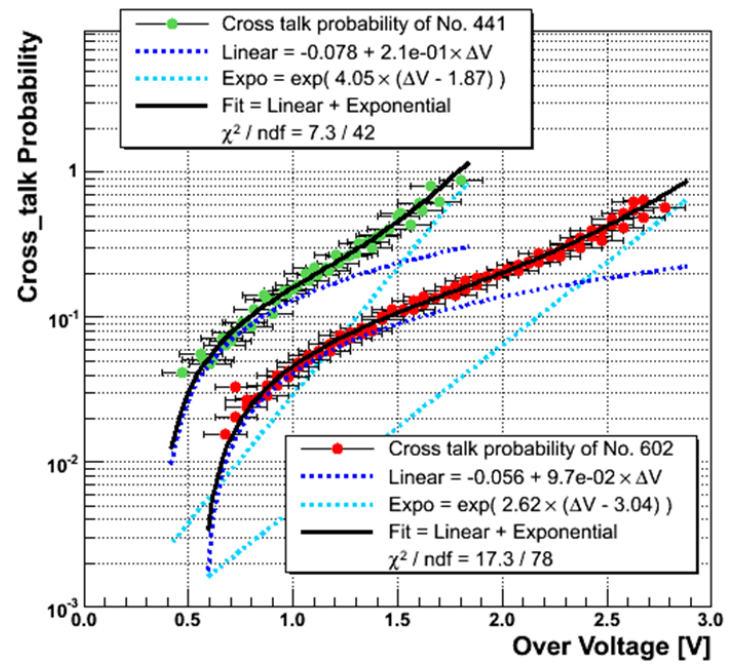

Figure 7: Over voltage characteristics of cross talk of $1 \mathrm{~mm} \times 1 \mathrm{~mm}$ size MPPCs with $-5^{\circ} \mathrm{C}, 5^{\circ} \mathrm{C}, 15^{\circ} \mathrm{C}$ and $25^{\circ} \mathrm{C}$, which can be fitted by combination of linear and exponential.

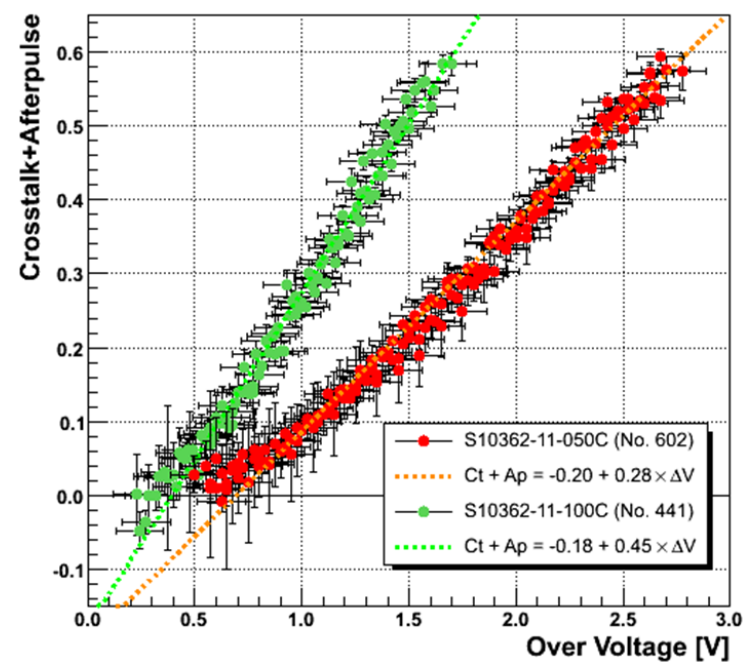

Figure 8: Over voltage characteristics of cross talk + after pulse probability of $1 \mathrm{~mm} \times 1 \mathrm{~mm}$ size MPPCs with $-5^{\circ} \mathrm{C}, 5^{\circ} \mathrm{C}, 15^{\circ} \mathrm{C}$ and $25^{\circ} \mathrm{C}$, and each device followed single line, respectively.

When low level light is illuminated on the MPPC, ADC counts distribution follows the Poisson distribution. Since the number of entry in the pedestal peak $N_{0}$ is not affected by the cross talk or after pulse, then the Poisson mean value $\lambda$ is expressed by $N_{0}$ and the number of all entry $N$, $\lambda=-\ln \frac{N_{0}}{N}$. If there is no cross talk and no after pulse, the expected number of entry at 1 p.e. peak $N_{1, \text { expected }}$ is $N_{1, \text { expected }}=N \lambda \mathrm{e}^{-\lambda}=N_{0} \ln \frac{N}{N_{0}}$. If the contamination due to cross talk and after pulse is in the measured entry of 1 p.e. peak $N_{1, \text { measured }}$, the probability of cross talk and after pulse $P_{C \& A}$ is estimated as $P_{C \& A}=1-\frac{N_{1, \text { expected }}}{N_{1, \text { measured }}}$. Estimated results of $P_{C \& A}$ of $1 \mathrm{~mm}$ size MPPCs are shown in Fig. [ 8 . The probability linearly increases with over voltage, and does not depend on the temperature, where the data measured at four different temperatures are plotted by the same symbol in this figure. So, at the fixed over voltage, the probability does not depend on the temperature as the cross talk probability does not.

\section{Summary}

We measured basic properties of PPDs that the voltage and temperature characteristics of gain, total noise rate, cross talk probability and cross talk + after pulse probability. We confirmed that the gain at fixed bias voltage strongly depends on the temperature. So we may have to use temperature compensation circuit for the future application. We found the cross talk probability as a function of over voltage is well fitted by the combination of the linear function and the exponential one. Also, we confirmed the characteristics of the gain of $3 \mathrm{~mm} \times 3 \mathrm{~mm}$ size MPPC is the same as those expected from $1 \mathrm{~mm} \times 1 \mathrm{~mm}$ size MPPC. 


\section{References}

[1] J. Buckley et al., 2008, arXiv:0810.0444v1

[2] G. Hermann et al., 2007, Proceedings of $30^{\text {th }}$ International Cosmic Ray Conference

[3] J. Haba, 2007, NIM A, 595, 154

[4] D. Renker et al., 2009, Journal of Instrumentation, 4, P04004 